\title{
The development of multidimensional screening model of geriatric syndrome for community- dwelling older adults: results of the Taiwan Integration Health and Welfare (TIHW) Study
}

Chi-Jung Tai

Pingtung Hospital

Tzyy-Guey Tseng

Kaohsiung Medical University Chung Ho Memorial Hospital

Ching-Ya Huang

Pingtung Hospital

Shu-Chuan Pan

Pingtung Hospital

Yu-Han Hsiao

Taichung Hospital

Yi-Hsin Yang

National Institute of Cancer Research

Meng-Chih Lee ( $\square$ mengchihlee@gmail.com )

Chung Shan Medical University

Research article

Keywords: Geriatric syndrome, prediction model, risk factors, multidimensional

Posted Date: October 14th, 2019

DOI: https://doi.org/10.21203/rs.2.15998/v1

License: (c) (1) This work is licensed under a Creative Commons Attribution 4.0 International License. Read Full License 


\section{Abstract}

Background: Comprehensive geriatric assessment (CGA) is a multidimensional and multidisciplinary diagnostic and treatment process that identifies geriatric syndrome in older adults. However, CGA program is not appropriate in community screening. To the best of our knowledge, there is no applicable multidimensional screening model to evaluate geriatric syndrome in community-dwelling older adults. This study aimed to identify the risk factors of geriatric syndrome among physical function tests, socioeconomic status, medical history, and healthy behaviours in community-dwelling older adults and develop a multidimensional prediction model for community screening.

Methods: A total of 1313 community-dwelling older adults aged 60 years or above were recruited from 58 communities in four aging cities in Taiwan. Geriatric syndrome was defined by disability using Instrumental Activities of Daily Livings, cognitive impairment using Short Portable Mental Status Questionnaire, depression using Geriatric Depression Scale, or by receiving mild disability card. The cutoff values of the physical function tests were calculated using receiver operating characteristic analysis. Multivariate logistic regression was used to evaluate the risk factors of geriatric syndrome, and the risk model was developed using stepwise logistic regression.

Results: We developed the new cutoff values in predicting geriatric syndrome for dominant handgrip strength test, 6-meter walk, and timed up-and-go tests, which were significantly associated with geriatric syndrome. Moreover, male sex, obesity, absence of labour activities, and participants who cannot report personal information, had depressive mood for the past 2 weeks, and a history of heart disease were associated with geriatric syndrome. Finally, we developed Taiwan Risk Scores for Geriatric Syndrome (TRSGS) with the cutoff value of 6 (sensitivity, $77.2 \%$; specificity, $75.5 \%$ ).

Conclusions: Most of the screening tools focus on specific problems such as sarcopenia, dementia, or frailty. The TRSGS model demonstrated a multidimensional prediction model, which could be applied in community screening for geriatric syndrome. Management of risk factors to prevent geriatric syndrome in the community is important.

\section{Background}

Geriatric syndrome is composed of multifactorial health problems resulting in multiple systems, which is common in older adults [1].The four independent predisposing factors for geriatric syndrome were older age, baseline cognitive impairment, baseline functional and sensory impairment, and impaired mobility, which often lead to an increased risk of poor health outcomes, including fall incidence, disability, hospitalisation, and mortality [2-4]. The prevalence of the geriatric syndrome with multimorbidity is about $10.4 \%$ [5]. The phenotype of geriatric syndrome includes frailty, sarcopenia, falls, weight loss, delirium, depression, dementia, urinary incontinence, oral problems, malnutrition, pressure ulcer, sexual dysfunction, visual loss, and functional impairment $[1,6,7]$. Management focusing on geriatric syndrome is effective in improving outcomes, but physicians often fail to recognise geriatric syndrome and 
appropriately perform therapeutic strategies $[8,9]$. This implies that we can stop this vicious circle by the early identification of geriatric syndrome and prevention of further disability.

Several screening tools have been developed and validated in predicting frailty, depression, dementia, or sarcopenia [10-12]. However, each model only predicted certain parts of the geriatric syndrome. In clinical practice, comprehensive geriatric assessment (CGA) is a multidimensional and multidisciplinary diagnostic and treatment process that identifies geriatric syndrome in older adults [13]. Moreover, a brief assessment tool was specifically designed for geriatric syndromes identification in general practice for general practitioners in the previous study [14]. The brief assessment tool was constructed, based on simple validated tests to detect each of these geriatric syndromes [15]. Additionally, a previous study also tried to use online geriatric assessment in acutely hospitalised patients [16]. However, these assessment tools were not appropriate in community screening. To the best of our knowledge, there is no applicable multidimensional screening model that predicts geriatric syndrome. Therefore, the present study aimed to develop an applicable multidimensional screening model for community-dwelling older adults in predicting geriatric syndrome.

\section{Methods}

\section{Community and participant enrolment}

To manage the health issues of the aging society in Taiwan [17], the Ministry of Taiwan supported the Taiwan Integration of Health and Welfare (TIHW) study to conduct a formal survey in 2017, which aimed to establish a screening strategy for geriatric syndrome in the community. First, of the 14 aging cities in Taiwan, 4 cities (Pingtung County, Tainan City, Changhua County, and Miaoli County) were selected from southern to northern part of Taiwan. Second, 7-14 communities in each city were selected, resulting in a total of 58 communities. Finally, the invitation (phone call and publicity leaflets) was sent to communitydwelling older adults ( $\geqq 60$ years) within the selected communities by the chief of the community. The participants were gathered in their local affiliated community centres for face-to-face interviews and assessments by trained investigators from Pingtung, Tainan, Changhua, or Miaoli Hospital, Ministry of Health and Welfare.

\section{Ethical approval and study protocol}

The protocol was approved by the Antai Medical Care Cooperation Antai-Tian-Sheng Memorial Hospital Institutional Review Board (Protocol Number: 17-030-B1; Approval Date: March 22, 2017). Before recruitment, all participants received a proper explanation about the study and provided informed consent for inclusion in the study. Participants who could read and write signed the written informed consents, and those who could not read and write impressed the name stamps or handprint with the assistance of their family members. 
The evaluation in the TIHW study comprised multi-dimensional questionnaires, physical function tests, and blood examinations. The TIHW study questionnaire (Approved Case No. 1060400299) was approved by the Directorate General of Budget, Accounting and Statistics, Executive Yuan, Taiwan, and was considered as a formal survey in Taiwan. The questionnaire included the survey of socioeconomic status, healthy behaviours, medical history, the Instrumental Activities of Daily Livings (IADLs), Short Portable Mental Status Questionnaire (SPMSQ), and Geriatric Depression Scale (GDS). The physical function tests included the dominant handgrip strength test, timed up-and-go (TUG) test, 6-meter walk test (6MWT), and five-time sit-to-stand test (FTSST). The fasting glucose, low-density lipoprotein cholesterol, high-density lipoprotein cholesterol, triglyceride, estimated glomerular filtration rate (eGFR), aspartate aminotransferase, and alanine aminotransferase tests were included in the blood examinations.

\section{Definition of geriatric syndrome and exclusion criteria}

Geriatric syndrome was defined by disability using IADLs, cognitive impairment using SPMSQ, depression using GDS or by receiving mild disability card. The mild disability card was given to patients with mild disability, such as mild cognitive impairment, low eye vision, or mild hearing impairment (auditory brainstem evoked response threshold between 55 and $69 \mathrm{~dB}$ ) [18]. We considered that IADLs, SPMSQ, GDS, and disability card could integratedly evaluate cognitive impairment, baseline functional and sensory impairment, and impaired mobility of geriatric syndrome. We excluded participants with a history of cerebrovascular disease, aged $<60$ years, and who received moderate to severe disability card.

The IADL scale is a valuable tool to evaluate older individuals with early-stage disease. In the current study, the IADL disability status was assessed with 5 activities from an IADL scale proposed by Lawton and Brody [19]. The sum of IADL score $\geqq 3$ was defined as IADL disability [20]. Cognitive function was measured using the nine-item SPMSQ with total scores ranging from 0 to 9 [21]. Participants with four or more errors were defined as having cognitive impairment [22]. Incident depression was considered to be present when the score of GDS-15 was greater than 5 [23].

\section{Questionnaires and physical function tests}

Questions related to socioeconomic status and medical history in the questionnaires aimed to collect data on participants' educational level, marital status, living status, medical history (hypertension, diabetes, liver disease, hyperlipidaemia, and chronic kidney disease), and socioeconomic status. Socioeconomic status was self-reported and was not based on real personal income. Moreover, the evaluation of healthy behaviours included exercise habits, smoking, and alcohol and areca nut consumption. Additionally, questions such as 'Do you have low interest in doing things for the past 2 weeks?' and 'Do you have depressive mood for the past 2 weeks?' were also evaluated. We also documented whether participants could remember their personal information correctly, including age, address, and telephone number. 
Physical performance was assessed through the dominant handgrip strength test, TUG test, 6MWT, and FTSST. Dominant handgrip strength was evaluated using a handheld dynamometry, and the result was expressed in kilogrammes (kg) [24]. The TUG test assesses transitional function, strength, agility, and dynamic balance [25]. In the TUG test, the participant was sitting with hands at starting position resting on the arms of the chair. The participants crossed the line, which was $3 \mathrm{~m}$ distant to the starting point, before turning around and walked back to sit down in the chair again. The participants were instructed to walk at their normal speed, and they performed one trial before they were timed. The timing of the TUG started when the participants' back came off the back of the chair and stopped when their buttocks touched the seat of the chair again [25]. The FTSST assesses functional lower extremity strength, transitional movements, balance, and fall risk [26]. The 6MWT was recommended as a functional assessment tool that predicts sarcopenia and fall $[11,27]$. The participants completed a six-meter walk measured in seconds at their normal walking speed. The study aimed to assess the validity and establish the cutoff values of the functional performance tests in predicting geriatric syndrome.

\section{Statistical analyses}

Descriptive statistics were calculated for the demographic characteristics of the participants. The receiver operating characteristic (ROC) curve and Youden's index were used to evaluate the diagnostic accuracy and to determine the cutoff values for handgrip strength, TUG test, FTSST, and 6MWT in predicting geriatric syndrome [28]. Multivariate regression models were used to investigate the risk factors of geriatric syndrome among physical function tests, socioeconomic status, healthy behaviours, and medical history. Risk scores were selected by stepwise logistic regression, and the validity of the prediction model was established by ROC analysis. All these analyses were performed using the Statistical Analysis System (SAS) version 9.4 (SAS Institute Inc., Cary, NC, USA).

\section{Results}

A total of 1449 participants were recruited in the TIHW study. We excluded 136 participants with a history of cerebrovascular disease, aged < 60 years, and who received moderate to severe disability card. Of the 1313 enrolled participants, 167 (12.7\%) and 1146 (87.3\%) were assigned to the geriatric syndrome group by our definition and the non-geriatric syndrome group, respectively. Participants in the geriatric syndrome group had older age, poorer educational level, higher rate of living with family (76.0\% vs. $64.6 \%)$, lesser exercise habits (47.3\% vs. $72.9 \%)$, more possibility to receive social assistance pension (10.8\% vs. 3.4\%), and higher rate of heart disease history than that of the non-geriatric syndrome group (Table 1). Moreover, participants in the geriatric syndrome group had a higher rate of depressive mood (14.4\% vs. 1.4\%), a lower interest in doing things (13.2\% vs. 1.0\%), and a poorer remembering of personal information (45.5\% vs. $18.5 \%$ ) than that of the non-geriatric syndrome group. Regarding physical function tests and blood examinations, the geriatric syndrome group had also poorer results in dominant handgrip strength test, 6MWT, TUG test, and FTSST and higher body mass index (BMI) $\left(26.5 \pm 4.7 \mathrm{vs} .24 .6 \pm 3.5 \mathrm{~kg} / \mathrm{m}^{2}\right)$ and lower 
eGFR $\left(75.1 \pm 20.9\right.$ vs. $\left.81.4 \pm 22.7 \mathrm{~mL} / \mathrm{min} / 1.73 \mathrm{~m}^{2}\right)$ compared to the non-geriatric syndrome group (Table 1).

\section{Cutoff values for physical function tests}

We developed the new cutoff values in predicting geriatric syndrome for dominant handgrip strength test, 6MWT, TUG test and FTSST. The dominant handgrip strength cutoff values were $27.3 \mathrm{~kg}$ (rea under the curve [AUC], 0.685) for men and $19.2 \mathrm{~kg}$ (AUC, 0.720) for women. The cutoff values of the 6MWT were $0.534 \mathrm{~m} / \mathrm{s}$ (AUC, 0.754$)$ for men and $0.465 \mathrm{~m} / \mathrm{s}$ (AUC, 0.735) for women. Moreover, the cutoff values of the TUG test were $12.8 \mathrm{~s}$ (AUC, 0.609) for men and $11.0 \mathrm{~s}$ (AUC, 0.677 ) for women. Additionally, the cutoff values for the FTSST were $10.0 \mathrm{~s}$ (AUC, 0.573) for men and $10.4 \mathrm{~s}$ (AUC, 0.650) for women. According to the ROC analysis, the 6MWT had the largest AUC among the tests. The figures associated with the statistics and cutoff values for each test are shown in Figure 1 and 2. We applied our cutoff values for each test in the further risk evaluation.

\section{Risk factors of geriatric syndrome}

Table 2 shows the adjusted odds ratio for each potential risk factor calculated by multivariate logistic regression. In a fully adjusted model, male, obesity, depressive mood for the past 2 weeks, poor remembering in personal information, absence of exercise habit, heart disease history, low handgrip strength, and abnormal 6MWT and TUG test were significantly associated with geriatric syndrome (Table 2). Living situation, marital status, age, self-reported economic status, educational level, and FTSST were not significantly associated with geriatric syndrome.

\section{Development of the Taiwan Risk Scores for Geriatric Syndrome (TRSGS) model}

Using stepwise logistic regression, 7 items were selected from the risk factors of geriatric syndrome, including 'cannot report personal information', 'depressive mood for the past 2 weeks', a history of heart disease, absence of labour activities, low handgrip strength, and abnormal 6MWT and TUG test. The weighting of each item was calculated by each adjusted odds ratio. Finally, the cutoff value of TRSGS model was 6 (AUC, 0.826; sensitivity, 77.2\%; specificity, 75.5\%) (Figure 3).

\section{Discussion}

To the best of our knowledge, there is no easily applicable screening tool in predicting geriatric syndrome in the community. Most of the screening tools focus on specific problems such as sarcopenia, dementia, or frailty $[29,30]$. The current study assessed the risk factors and developed a multidimensional risk model in predicting geriatric syndrome in community-dwelling older adults. We found out that TRSGS 
score $\geqq 6$ was significantly associated with geriatric syndrome, which comprised items to evaluate cognitive impairment, depressive disorder, baseline functional and sensory impairment, and impaired mobility in community-dwelling older adults. Therefore, the TRSGS can be used in predicting geriatric syndrome for community-dwelling older adults. Further cohort study will be designed to validate the predictors of geriatric syndrome and support the implementation of preventive strategies in communitydwelling older adults.

In the previous studies, physical function tests were considered as indicators for sarcopenia or frailty [11]. For example, low handgrip strength was defined as $<26 \mathrm{~kg}$ for men and $<18 \mathrm{~kg}$ for women by the Asian Working Group for Sarcopenia (AWGS) in the screening of sarcopenia. The suggested cutoff values ( $<27.3 \mathrm{~kg}$ for men and $<19.2 \mathrm{~kg}$ for women) in the current study were higher than that of the previous studies. Using the cutoff value suggested by the AWGS might decrease the sensitivity and increase the specificity because sarcopenia is only part of the geriatric syndrome (Figure $1 \mathrm{~A}$ and $2 \mathrm{~A}$ ). Therefore, we established the new cutoff values for the physical function tests in predicting geriatric syndrome. The multiple physical function tests evaluated different parts of the mobility function. Finally, the discriminant abilities of the dominant handgrip strength test, 6MWT, and TUG test were associated with geriatric syndrome.

The incidence rate of depression in older adults was reported to be underestimated in a previous study [31]. The UK National Institute for Health and Clinical Excellence recommended two screening questions such as 'During the past month, have you often been bothered by feeling down, depressed, or hopeless?' and 'During the past month, have you often been bothered by having little interest or pleasure in doing things?' for older adults for depression screening [32]. Therefore, two questions such as 'Do you have low interest in doing things for the past 2 weeks?' and 'Do you have depressive mood for the past 2 weeks?' were included in the annual free preventive health examination for older adults aged 65 years or older in Taiwan for depression screening. In the current study, both questions were proven to be significant risk factors associated with geriatric syndrome, where depression was a key component. Using stepwise logistic regression, the question 'Do you have depressive mood for the past 2 weeks?' was selected by the TRSGS model. Similarly, several developed cognitive screening tools to evaluate dementia in the community-dwelling older adults, such as the Ascertain Dementia 8-item Informant Questionnaire, were available [33]. We tried to use one screening item to evaluate cognitive function in the current study. We have proven that if participants could not report their personal information, they were significantly associated with geriatric syndrome in our study. We assumed that a simple item can be a good predictor for the screening of cognitive impairment in community-dwelling older adults.

Our study has some limitations. First, our study was a cross-sectional study that reported the association between risk factors and geriatric syndrome. The incidence of geriatric syndrome cannot be estimated. Second, although we recruited participants from 58 communities among 4 cities, the generalisation of the results in this study is still questionable. Third, the records of socioeconomic status, medical history, and healthy behaviours were self-reported and self-recalled, which may have bias. Finally, although we 
included several covariates associated with geriatric syndrome, there may be other potential confounders that were unavailable.

\section{Conclusions}

Geriatric syndrome is a complex health status associated with multiple domains and dimensions. Multimorbidity poses profound challenges to older adults, and it is important to immediately manage these risk factors [34]. Therefore, it is important to develop a multidimensional screening model for community-dwelling older adults in predicting geriatric syndrome. The current study determined the multiple risks factors of geriatric syndrome. Moreover, we developed the TRSGS model demonstrating a multidimensional prediction model with high sensitivity and specificity in community screening. The current study has provided valuable insights for further studies on the development of multidimensional screening models.

\section{Abbreviations}

6MWT: 6-meter walk test; AUC: area under the curve; AWGS: Asian Working Group for Sarcopenia; CGA: Comprehensive geriatric assessment; eGFR: Estimated glomerular filtration rate; FTSST: five-time sit-tostand test; GDS: Geriatric Depression Scale; IADLs: Instrumental Activities of Daily Livings; Kg: kilogrammes; OR: Odds ratio; ROC: receiver operating characteristic; SAS: Statistical Analysis System; SPMSQ: Short Portable Mental Status Questionnaire; TIHW: Taiwan Integration Health and Welfare; TRSGS: Taiwan Risk Scores for Geriatric Syndrome; TUG: timed up-and-go

\section{Declarations}

\section{Ethics approval and consent to participate}

The study protocol was approved by the Antai Medical Care Cooperation Antai-Tian-Sheng Memorial Hospital Institutional Review Board. Before recruitment, all participants received a proper explanation about the study and provided informed consent for inclusion in the study.

\section{Consent for publication}

Not applicable.

\section{Availability of data and materials}

The datasets used and analysed during the current study are not publicity available, but are available from the corresponding author on reasonable request with the permission of the Ministry of Health and Welfare, Taiwan. 


\section{Competing interests}

The authors declare that they have no competing interests.

\section{Funding}

This work was supported by the Ministry of Health and Welfare, Taiwan (Grant number: M06M2346). The study sponsor participated in the study design, but played no role in participant recruitment, data analysis, or paper preparation.

\section{Author's contributions}

All authors approved the submitted version of the manuscript, and ensured the accuracy and integrity of this work. CJT and MCL initiated and designed the study. CYH, SCP and YHH contributed to data acquisition. CJT and YHY performed data analysis. CJT, TGT, YHY and MCL drafted the manuscript and substantively revised it.

\section{Acknowledgements}

We thank our colleagues from Pingtung Hospital, Tainan Hospital, Changhua Hospital, and Miaoli Hospital, Ministry of Health and Welfare, Taiwan, for their support in completing the survey. We also thank the statistical experts from Directorate-General of Budget, Accounting and Statistics, Executive Yuan, Taiwan.

\section{References}

1.Flacker JM: What is a geriatric syndrome anyway? J Am Geriatr Soc 2003, 51(4):574-576.

2.Inouye SK, Studenski S, Tinetti ME, Kuchel GA: Geriatric syndromes: clinical, research, and policy implications of a core geriatric concept. J Am Geriatr Soc 2007, 55(5):780-791.

3.Tinetti ME, Inouye SK, Gill TM, Doucette JT: Shared risk factors for falls, incontinence, and functional dependence. Unifying the approach to geriatric syndromes. Jama 1995, 273(17):1348-1353.

4.Wang SY, Shamliyan TA, Talley KM, Ramakrishnan R, Kane RL: Not just specific diseases: systematic review of the association of geriatric syndromes with hospitalization or nursing home admission. Archives of gerontology and geriatrics 2013, 57(1):16-26.

5.Cheung JTK, Yu R, Wu Z, Wong SYS, Woo J: Geriatric syndromes, multimorbidity, and disability overlap and increase healthcare use among older Chinese. BMC Geriatrics 2018, 18(1):147. 
6.Fried LP, Tangen CM, Walston J, Newman AB, Hirsch C, Gottdiener J, Seeman T, Tracy R, Kop WJ, Burke $\mathrm{G}$ et al: Frailty in older adults: evidence for a phenotype. The journals of gerontology Series A, Biological sciences and medical sciences 2001, 56(3):M146-156.

7.Morley JE: The Importance of Geriatric Syndromes. Mo Med 2017, 114(2):99-100.

8.Stuck AE, Siu AL, Wieland GD, Adams J, Rubenstein LZ: Comprehensive geriatric assessment: a metaanalysis of controlled trials. Lancet 1993, 342(8878):1032-1036.

9.Ng TP, Feng L, Nyunt MS, Feng L, Niti M, Tan BY, Chan G, Khoo SA, Chan SM, Yap P et al: Nutritional, Physical, Cognitive, and Combination Interventions and Frailty Reversal Among Older Adults: A Randomized Controlled Trial. Am J Med 2015, 128(11):1225-1236.e1221.

10.Thompson MQ, Theou O, Tucker GR, Adams RJ, Visvanathan R: Recurrent Measurement of Frailty Is Important for Mortality Prediction: Findings from the North West Adelaide Health Study. J Am Geriatr Soc 2019.

11.Chen LK, Liu LK, Woo J, Assantachai P, Auyeung TW, Bahyah KS, Chou MY, Chen LY, Hsu PS, Krairit O et al: Sarcopenia in Asia: consensus report of the Asian Working Group for Sarcopenia. Journal of the American Medical Directors Association 2014, 15(2):95-101.

12.Ishizaki T, Watanabe S, Suzuki T, Shibata $\mathrm{H}$, Haga $\mathrm{H}$ : Predictors for functional decline among nondisabled older Japanese living in a community during a 3-year follow-up. J Am Geriatr Soc 2000, 48(11):1424-1429.

13.Pilotto A, Cella A, Pilotto A, Daragjati J, Veronese N, Musacchio C, Mello AM, Logroscino G, Padovani A, Prete $\mathrm{C}$ et al: Three Decades of Comprehensive Geriatric Assessment: Evidence Coming From Different Healthcare Settings and Specific Clinical Conditions. Journal of the American Medical Directors Association 2017, 18(2):192.e191-192.e111.

14.Senn N, Monod S: Development of a Comprehensive Approach for the Early Diagnosis of Geriatric Syndromes in General Practice. Frontiers in medicine 2015, 2:78.

15.Mueller YK, Monod S, Locatelli I, Büla C, Cornuz J, Senn N: Performance of a brief geriatric evaluation compared to a comprehensive geriatric assessment for detection of geriatric syndromes in family medicine: a prospective diagnostic study. BMC Geriatrics 2018, 18(1):72.

16.Gray L, Dakin L, Counsell S, Edwards H, Wootton R, Martin-Khan M: 'Online' geriatric assessment procedure for older adults referred for geriatric assessment during an acute care episode for consideration of reliability of triage decisions. BMC Geriatrics 2012, 12(1):10.

17.Lin Y-Y, Huang C-S: Aging in Taiwan: Building a Society for Active Aging and Aging in Place. The Gerontologist 2015, 56(2):176-183. 
18.The regulation and management of disability card [https://dep.mohw.gov.tw/DONAHC/cp-10325186-104.html]

19.Lawton MP, Brody EM: Assessment of older people: self-maintaining and instrumental activities of daily living. The Gerontologist 1969, 9(3):179-186.

20.Chiu CJ, Wray LA, Ofstedal MB: Diabetes-related change in physical disability from midlife to older adulthood: evidence from 1996-2003 Survey of Health and Living Status of the Elderly in Taiwan.

Diabetes Res Clin Pract 2011, 91(3):413-423.

21.Pfeiffer E: A short portable mental status questionnaire for the assessment of organic brain deficit in elderly patients. J Am Geriatr Soc 1975, 23(10):433-441.

22.Alley DE, Crimmins EM, Karlamangla A, Hu P, Seeman TE: Inflammation and rate of cognitive change in high-functioning older adults. The journals of gerontology Series A, Biological sciences and medical sciences 2008, 63(1):50-55.

23.Peters R, Pinto E, Beckett N, Swift C, Potter J, McCormack T, Nunes M, Grimley-Evans J, Fletcher A, Bulpitt C: Association of depression with subsequent mortality, cardiovascular morbidity and incident dementia in people aged 80 and over and suffering from hypertension. Data from the Hypertension in the Very Elderly Trial (HYVET). Age and Ageing 2010, 39(4):439-445.

24.Paul DJ, Nassis GP: Testing strength and power in soccer players: the application of conventional and traditional methods of assessment. J Strength Cond Res 2015, 29(6):1748-1758.

25.Nordin E, Lindelöf N, Rosendahl E, Jensen J, Lundin-olsson L: Prognostic validity of the Timed Up-andGo test, a modified Get-Up-and-Go test, staff's global judgement and fall history in evaluating fall risk in residential care facilities. Age and Ageing 2008, 37(4):442-448.

26.Tap L, Boyé NDA, Hartholt KA, van der Cammen TJM, Mattace-Raso FUS: Association of estimated glomerular filtration rate with muscle function in older persons who have fallen. Age and Ageing 2017, 47(2):269-274.

27.Tiedemann A, Shimada H, Sherrington C, Murray S, Lord S: The comparative ability of eight functional mobility tests for predicting falls in community-dwelling older people. Age and Ageing 2008, 37(4):430435.

28.Ruopp MD, Perkins NJ, Whitcomb BW, Schisterman EF: Youden Index and optimal cut-point estimated from observations affected by a lower limit of detection. Biometrical journal Biometrische Zeitschrift 2008, 50(3):419-430.

29.Yamada M, Nishiguchi S, Fukutani N, Tanigawa T, Yukutake T, Kayama H, Aoyama T, Arai H: Prevalence of sarcopenia in community-dwelling Japanese older adults. Journal of the American Medical Directors Association 2013, 14(12):911-915. 

IDENTIFYING FRAILTY IN COMMUNITY DWELLING OLDER PEOPLE: A SYSTEMATIC REVIEW. Age and Ageing 2014, 43(suppl_2):ii10-ii11.

31.Weyerer S, Eifflaender-Gorfer S, Wiese B, Luppa M, Pentzek M, Bickel H, Bachmann C, Scherer M, Maier W, Riedel-Heller SG: Incidence and predictors of depression in non-demented primary care attenders aged 75 years and older: results from a 3-year follow-up study. Age and Ageing 2013, 42(2):173-180.

32.Esiwe C, Baillon S, Rajkonwar A, Lindesay J, Lo N, Dennis M: Screening for depression in older adults on an acute medical ward: the validity of NICE guidance in using two questions. Age and Ageing 2015, 44(5):771-775.

33.Mao H-F, Chang L-H, Tsai AY-J, Huang W-NW, Tang L-Y, Lee H-J, Sun Y, Chen T-F, Lin K-N, Wang P-N et al: Diagnostic accuracy of Instrumental Activities of Daily Living for dementia in community-dwelling older adults. Age and Ageing 2018, 47(4):551-557.

34.Lawrence V: Independence and the pernicious threat of multimorbidity. Age and Ageing 2019, 48(4):472-473.

\section{Tables}


Table 1 Characteristics of the Participants $(\mathrm{N}=1313)$

Geriatric syndrome

\begin{tabular}{lccc} 
& Yes, $\mathrm{n}=$ & No, $\mathrm{n}=$ & $\begin{array}{c}P \text { - } \\
167\end{array}$ \\
\hline Age $^{*}$ & $74.3 \pm 7.8$ & $71.8 \pm 6.7$ & $<.001^{\dagger}$ \\
Female, $\mathrm{v}(\%)$ & $86(51.5)$ & $629(54.9)$ & 0.41 \\
\hline Educational level, $\mathrm{n}(\%)$ & & & $0.002^{\ddagger}$ \\
\hline Uneducated & & & \\
\hline Elementary school & $29(17.4)$ & $142(12.4)$ & \\
\hline Junior high school & $81(48.5)$ & $426(37.2)$ & \\
\hline Senior high school & $20(12.0)$ & $162(14.1)$ & \\
\hline College or above & $19(11.4)$ & $220(19.2)$ & \\
\hline
\end{tabular}

Marital status, n (\%)

0.81

Married and cohabitant

$25(15.0) \quad 180(15.7)$

Single and divorced and widowed

$142(85.0) \quad 966(84.3)$

Living condition, n (\%)

$0.004^{\ddagger}$

Living alone

Living with spouse

Living with children

Self-reported economic status, n (\%)
$40(24.0) \quad 405(35.3)$

$15(9.0) \quad 131(11.4)$

$112(67.0) \quad 610(53.2)$ 
High-upper income

Middle income

Low income

Social assistance pension, $\mathrm{n}(\%)$

Smoking, n (\%)

No

$<1$ pack per day (PPD)

$\geqq 1 \mathrm{PPD}$

Alcohol consumption, n (\%)

No

Social drinking

Alcoholism

Areca nuts consumption, n (\%)

Exercise habits, n (\%)

No

Domestic labour, farming

Exercise $\geqq 2.5 \mathrm{hrs} /$ week

Self-reported medical history, n (\%)

Hypertension

Diabetes mellitus
$9(5.4)$

$33(2.9)$

$82(49.1) \quad 458(40.0)$

$76(45.5)$

18 (10.8)

$39(3.4)$

$<.001^{\ddagger}$

$160(95.8) \quad 1080(94.2)$

$4(2.4)$

$47(4.1)$

$3(1.8)$

$19(1.7)$

\subsection{6}

$0.005^{\ddagger}$
$152(91.0) \quad 990(86.4)$

$10(6.0) \quad 145(12.7)$

$5(3.0)$

$11(1.0)$

$2(1.2)$

$15(1.3)$

0.91
$88(52.7) \quad 310(27.1)$

$71(42.5) \quad 518(45.2)$

$8(4.8) \quad 318(27.8)$ 
Heart disease

Hyperlipidaemia

Chronic kidney disease

Depressive mood for the past 2 weeks, n (\%)

Low interest of doing things for the past 2 weeks, $n$ (\%)

Cannot remember personal information, n (\%)

Dominant handgrip strength, $\mathrm{kg}(\mathrm{n}=1305)^{*}$

6-meter walk test, $\mathrm{m} / \mathrm{s}(\mathrm{n}=1160)^{*}$

Five-time sit-to-stand test, sec $(\mathrm{n}=1290)^{*}$

Timed up-and-go test, sec $(\mathrm{n}=1293)^{*}$

Physical and blood examinations $(\mathrm{n}=973)^{*}$
$35(21.0)$

$7(4.2)$

$3(1.8)$

$24(14.4)$

$22(13.2)$ $101(8.8)<.001^{\ddagger}$

$58(5.1) \quad 0.63$

$15(1.3) \quad 0.61$

$76(45.5) \quad 212(18.5) \quad<.001^{\ddagger}$

$22.2 \pm 8.4 \quad 26.8 \pm 10.8<.001^{\dagger}$

$0.45 \pm 0.18$

$0.62 \pm 0.23<.001^{\dagger}$

$12.2 \pm 6.9 \quad 10.2 \pm 18.5 \quad 0.01^{\dagger}$

$13.4 \pm 8.0 \quad 10.1 \pm 3.6<.001^{\dagger}$
BMI, $\mathrm{kg} / \mathrm{m}^{2}$

Fasting glucose level, mg/dL

Aspartate aminotransferase, IU/L

Alanine aminotransferase, IU/L

Low-density lipoprotein cholesterol, mg/dL

High-density lipoprotein cholesterol, mg/dL

Triglyceride, $\mathrm{mg} / \mathrm{dL}$
$26.5 \pm 4.7 \quad 24.6 \pm 3.5$

$0.005^{\dagger}$

$107.3 \pm 34.3 \quad 102.7 \pm 25.6 \quad 0.33$

$24.4 \pm 8.8 \quad 25.4 \pm 11.7 \quad 0.43$

$22.2 \pm 11.0 \quad 23.2 \pm 15.7 \quad 0.54$

$110.6 \pm 29.5 \quad 116.1 \pm 33.2 \quad 0.23$

$54.5 \pm 16.9 \quad 57.7 \pm 16.7$

0.17

$121.8 \pm 68.8 \quad 116.7 \pm 73.2 \quad 0.62$ 
eGFR, $\mathrm{mL} / \mathrm{min} / 1.73 \mathrm{~m}^{2}$

${ }^{*}$ Values are given as mean \pm standard deviation, not no. (\%)

${ }^{\dagger}$ T-test, $p<0.05 ;{ }^{\ddagger}$ Chi-square test, $p<0.05$

BMI, body mass index; eGFR, estimated glomerular filtration rate 
Table 2 Multivariate logistic regression model in predicting geriatric syndrome

\begin{tabular}{|c|c|c|}
\hline Variables & Adjusted OR (95\% CI) & $p$-value \\
\hline Male & $1.74(1.11-2.74)$ & $0.02^{*}$ \\
\hline Age $(65<$ age $\leqq 70)$ & $0.96(0.52-1.75)$ & 0.89 \\
\hline Age $(70<$ age $\leqq 75)$ & $1.31(0.71-2.42)$ & 0.38 \\
\hline Age ( $75<$ age $)$ & $1.73(0.99-3.01)$ & 0.06 \\
\hline Educational level (elementary school or below) & $0.93(0.53-1.64)$ & 0.80 \\
\hline Single, divorced, or widowed & $0.76(0.41-1.42)$ & 0.40 \\
\hline Living alone & $0.72(0.34-1.55)$ & 0.40 \\
\hline Self-reported low income & $1.07(0.37-3.11)$ & 0.90 \\
\hline Social assistance pension & $1.52(0.66-3.50)$ & 0.33 \\
\hline Obesity $\left(\mathrm{BMI} \geqq 27 \mathrm{~kg} / \mathrm{m}^{2}\right)$ & $2.62(1.18-5.81)$ & $0.02^{*}$ \\
\hline Overweight $\left(24 \leqq \mathrm{BMI}<27 \mathrm{~kg} / \mathrm{m}^{2}\right)$ & $1.17(0.50-2.72)$ & 0.72 \\
\hline Underweight $\left(\mathrm{BMI}<18.5 \mathrm{~kg} / \mathrm{m}^{2}\right)$ & $0.88(0.10-7.57)$ & 0.91 \\
\hline Smoking & $0.56(0.20-1.60)$ & 0.28 \\
\hline Alcohol consumption & $0.62(0.29-1.29)$ & 0.20 \\
\hline Areca nut consumption & $3.24(0.59-17.8)$ & 0.18 \\
\hline Depressed mood for the past 2 weeks & $3.78(1.06-13.5)$ & $0.04^{*}$ \\
\hline Low interest in doing things for the past 2 weeks & $3.31(0.81-13.6)$ & 0.10 \\
\hline Cannot remember personal information & $2.80(1.82-4.31)$ & $<0.001^{*}$ \\
\hline Absence of exercise habit & $1.59(1.06-2.40)$ & $0.03^{*}$ \\
\hline Domestic labour or farming & $0.23(0.10-0.52)$ & $<0.001^{*}$ \\
\hline \multicolumn{3}{|l|}{ Self-reported medical history } \\
\hline Heart disease & $2.47(1.41-4.33)$ & $0.002^{*}$ \\
\hline Hypertension & $1.30(0.86-1.96)$ & 0.22 \\
\hline Diabetes mellitus & $0.60(0.33-1.11)$ & 0.10 \\
\hline Chronic kidney disease & $0.92(0.23-3.67)$ & 0.91 \\
\hline Hyperlipidaemia & $0.60(0.20-1.76)$ & 0.35 \\
\hline Low handgrip strength & $2.54(1.66-3.88)$ & $<0.001^{*}$ \\
\hline
\end{tabular}


Abnormal lower extremity function tests

\begin{tabular}{lcc}
\hline 6MWT & $2.55(1.63-3.98)$ & $<0.001^{*}$ \\
\hline TUG test & $2.11(1.30-3.43)$ & $0.003^{*}$ \\
\hline FTSST & $0.99(0.95-1.03)$ & 0.51 \\
\hline
\end{tabular}

6MWT, 6-meter walk test; CI, confidence interval; OR, odds ratio; TUG test, timed up-andgo test

${ }^{*}$ Multivariate logistic regression, $p$-value $<0.05$

\section{Figures}
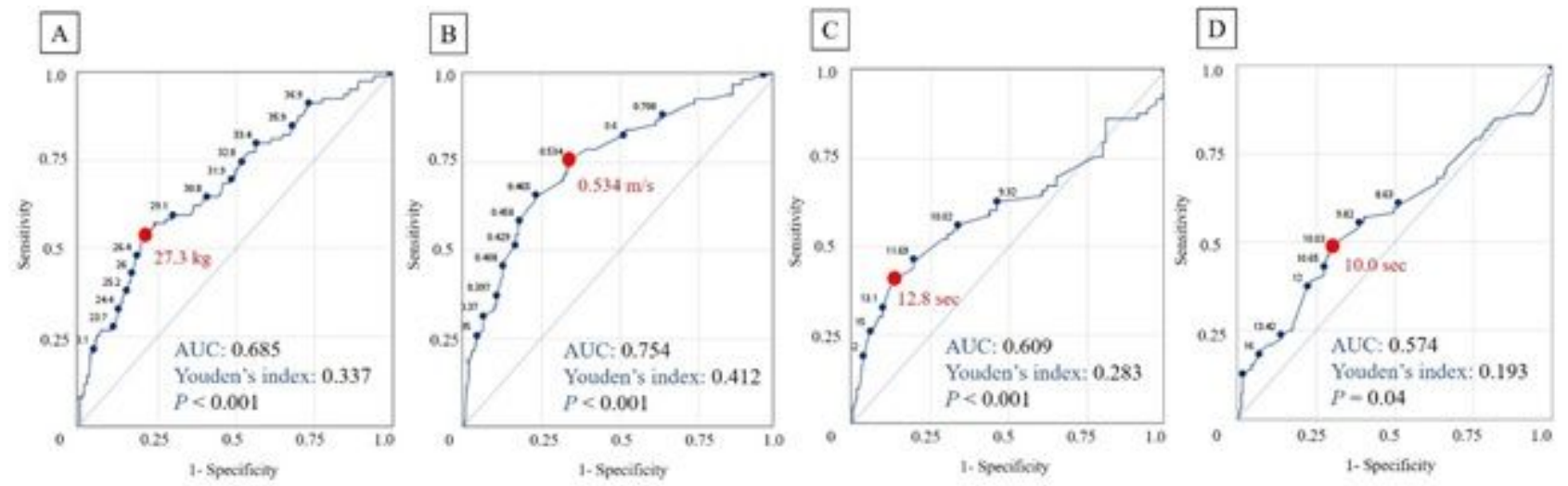

Figure 1

Receiver operating characteristic curve of physical function tests in male participants in prediction of geriatric syndrome (A) Dominant handgrip strength (B) 6-meter walk test (C) timed up-and-go test (D) Five-time-sit-to-stand test.
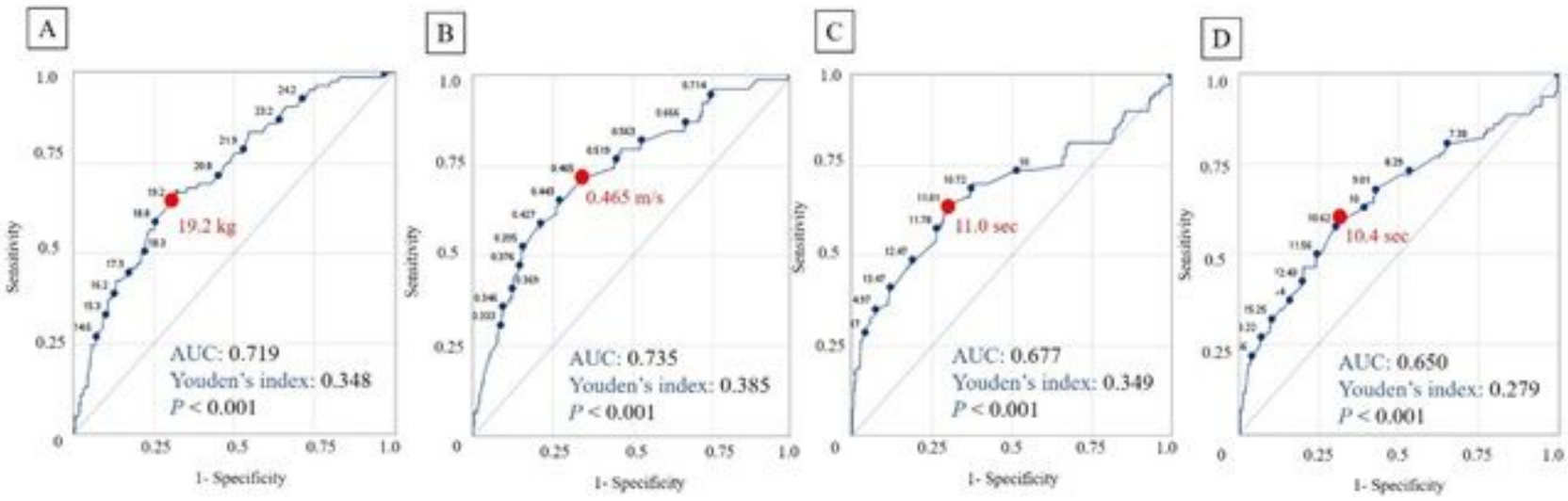

Figure 2 
Receiver operating characteristic curve of physical function tests in female participants in prediction of geriatric syndrome (A) Dominant handgrip strength (B) 6-meter walk test (C) timed up-and-go test (D) Five-time-sit-to-stand test.

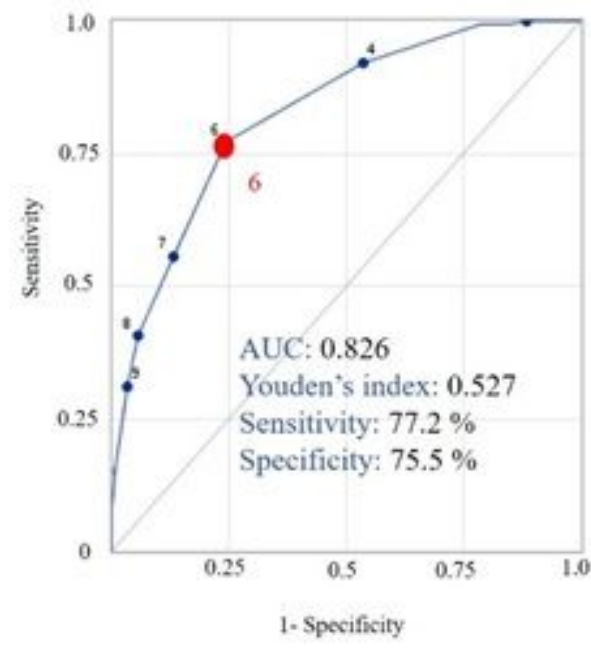

\begin{tabular}{cccc}
\multicolumn{4}{c}{ Stepwise Logistic Regression } \\
\hline Variables & $\begin{array}{c}\text { Odds ratio } \\
(\mathbf{9 5 \%} \text { CI) }\end{array}$ & P value & $\begin{array}{c}\text { Risk score } \\
\text { weighting }\end{array}$ \\
\hline $\begin{array}{c}\text { Cannot report } \\
\text { personal information }\end{array}$ & $2.62(1.74-3.95)$ & $<.001$ & 2 \\
\hline Depressive mood & $8.23(3.75-18.1)$ & $<.001$ & 5 \\
\hline Heart disease & $2.14(1.25-3.66)$ & 0.006 & 1 \\
\hline $\begin{array}{c}\text { Absence of labour } \\
\text { activities }\end{array}$ & $4.33(1.92-9.80)$ & $<.001$ & 3 \\
\hline Low handgrip & $2.31(1.55-3.43)$ & $<.001$ & 1 \\
\hline 6MWT & $2.64(1.71-4.07)$ & $<.001$ & 2 \\
\hline TUG test & $1.73(1.14-2.62)$ & 0.01 & 1 \\
\hline
\end{tabular}

Figure 3

The validity of the Taiwan Risk Scores for Geriatric Syndrome model. 6MWT, 6-meter walk test; TUG test, timed up-and-go test. 